\title{
Facile Fabrication of Composite Scaffolds for Long-Term Controlled Dual Drug Release
}

\author{
Dawei Li, ${ }^{1}$ Chao Li, ${ }^{1}$ Xing Wang $\mathbb{D}^{2,3}$ Chunlin Li, ${ }^{1}$ Tunan Sun, ${ }^{1}$ Jin Zhou $\mathbb{D}^{1},{ }^{1}$ and Gang Li $\mathbb{D}^{1}$ \\ ${ }^{1}$ The 8th Medical Center of Chinese, PLA General Hospital, Beijing 100091, China \\ ${ }^{2}$ Beijing National Laboratory for Molecular Sciences, State Key Laboratory of Polymer Physics \& Chemistry, Institute of Chemistry, \\ Chinese Academy of Sciences, Beijing 100190, China \\ ${ }^{3}$ University of Chinese Academy of Sciences, Beijing 100049, China
}

Correspondence should be addressed to Xing Wang; wangxing@iccas.ac.cn, Jin Zhou; huoshan1975@sina.com, and Gang Li; ligamg@sina.com

Received 14 June 2019; Accepted 12 September 2019; Published 5 January 2020

Guest Editor: Xiaosong Cao

Copyright ( 92020 Dawei Li et al. This is an open access article distributed under the Creative Commons Attribution License, which permits unrestricted use, distribution, and reproduction in any medium, provided the original work is properly cited.

\begin{abstract}
Bone tuberculosis (TB) caused by mycobacterium tuberculosis continues to present a formidable challenge to humans. To effectively cure serious bone $\mathrm{TB}$, a novel kind of composite scaffolds with long-term dual drug release behaviours were prepared to satisfy the needs of both bone regeneration and antituberculosis drug therapy. In virtue of an improved $\mathrm{O} / \mathrm{W}$ emulsion technique, water-soluble isoniazid (INH)-loaded gelatin microparticles were obtained by tailoring the content of $\beta$-tricalcium phosphate $(\beta$-TCP), which played significant roles in INH entrapment efficiency and drug release behaviours. By mixing with the poly( $\varepsilon$-caprolactone)-blockpoly (lactic-co-glycolic acid) ( $b$-PLGC) solution containing oil-soluble rifampicin (RFP) via the particle leaching combined with phase separation technique, the dual drugs-loaded composite scaffolds were fabricated, which possessed interconnected porous structures and achieved the steady release of INH and RFP drugs for three months. Moreover, this dual drugs-loaded system could basically achieve their expectant roles of respective drugs without obvious influences with each other. This strategy on preparation of intelligent composite scaffolds with the multi-drugs loading capacity and controlled long-term release behaviour will be potential and promising substrates in clinical treatment of bone tuberculosis.
\end{abstract}

\section{Introduction}

Bacterial infections are one of the most common diseases threatening the human health, which have resulted in the severe illness to destroy the infected tissues, such as bone tuberculosis (TB) and osteomyelitis [1-4]. Traditional therapy of the infections mainly includes the disinfection/bacteriostasis and reparation of the destroyed tissues. Take serious bone TB for instance, the standard course involves a more-than-6months medication treatment to inhibit bacteria and a bone implantation after the lesion removed operation to fill and repair the cavities. A local drug delivery system (DDS) offers an effective approach for the drug therapy of bone TB. It possesses advantages of specific site delivery, drug dosage optimization and release duration. Therefore, DDS technology is recognized as the best candidate for achieving the drug therapy while tissue engineering is the most prospective method for the defect reparation [5-9]. It follows that the combination of DDS and tissue engineering should be a natural way to simultaneously satisfy the two aspects of bone TB therapy.

Recently, many researchers have been focusing on this feasible strategy. For example, Huang's groups and Kang's groups had utilized a chemical bonding technique to conjugate the drugs to biodegradable polymers to gain the tissue engineering scaffolds respectively, which could implement the prolonged drug release and satisfactory tissue regeneration. However, the chemical bonding technique always carried out a long-term drug release (about 6 months) with a slightly initial burst release. In addition, it had inevitable defectiveness of the possible drug degeneration and incomplete drug release, in particular the possible change in pharmacological effects after chemical bonding and during degradation of the polymer. When its molecular weight is lower than 5000, the degraded fragment of polylactone linked with a drug molecule can be dissolved in water and is considered as a drug molecule, but whether it can play the same role as a drug molecule is 
doubtful. As a result, the physical blending technique was more preferable for achieving the controlled drug release from scaffolds [10-14]. Then, a newly designed polylactone of $b$-PLGC was adopted to produce a bone tissue-engineered scaffold with capability of elongating the drug release time to 98 days and reducing the initial burst release to lower than $40 \%$. Nevertheless, it was mostly reported that with similar physical blending technique the release time could only last less than 50 days and the initial burst release reached up to higher than $60 \%$ [15]. In spite of technical differences, the above-mentioned works can meet the demands of both reparative and bacteriostatic functions. Nonetheless, the constant release of only one drug in the focus of infection may lead to the drug-resistance of bacteria; in this case, it is urgent to adopt the multiple drugs for local therapy in clinical practice [16, 17]. Therefore, establishment of long-term multiple drug release system is a correct direction to move forward not only in this research but in clinical area.

In addition to the first-line antituberculosis drugs such as isoniazid (INH), rifampicin (RFP), pyrazinamide and ethambutol, others like $\mathrm{p}$-aminosalicylic acid, ethionamide, protionamide and cycloserine are also included for the multiple-drugs therapy of bone TB [18-23]. These drugs can be classified by solubility into two categories of water-soluble and oil-soluble. For the purpose of simplifying the laboratory model, one typical drug from each category was picked out to be loaded in tissue engineering scaffolds at the same time. Considering the clinically wide usage, INH was the suitable representative of water-soluble drugs and RFP was that of oil-soluble drugs. It was easy to load RFP into the tissue engineering scaffolds by the physical blending technique because the oil-soluble RFP could dissolve well in organic solvents and disperse evenly in the polylactone scaffold. Oppositely, the loading of INH was difficult. The phase separation of INH and polylactone may result in an uneven INH distribution. In addition, the water-solubility of INH demands a non-aqueous washing process to avoid uncontrolled loss, but most commonly, tissue-engineering scaffolds fabricating technique was solution cast/ porogen leaching, in which the inorganic salts (mostly $\mathrm{NaCl}$ particles) were adopted as porogen while the water was a necessity during leaching process [24-26]. To circumvent this problem, gelatin coating and crosslinking techniques were taken to load INH into TCP nanoparticles. Under this circumstance, the particles could be carried into polylactone/TCP composite scaffolds with little INH loss after porogen leaching process.

In this work, we incorporated the TCP nanoparticles into the RFP-containing PLGC polymer matrix to form the composite scaffolds. Since PCL has high permeability for drug molecules and PLGA has flexibility in degradation adjustment, the RFP-releasing behavior and degradation rate of scaffolds were mainly controlled by the degradation and drug permeability of $b$-PLGC polymer, which could effectively improve the uniformity, extend the release duration and suppress the initial burst release. Furthermore, by means of the complicated physical blending technique, the water-soluble INH drug was also encapsulated into the composite scaffolds to yield a novel kind of dual anti-tuberculosis drug-loaded composite scaffold and realize a controllably prolonged local drug delivery. Using this principle, many sophisticated scaffold materials with the multiple loading and tailored releases of drugs may have great applications in bone TB therapy.

\section{Materials and Methods}

2.1. Materials. Lactide and glycolide were purchased from Purac (Netherlands) and purified by recrystallization in ethyl acetate twice. $\varepsilon$-caprolactone was purchased from Acros Chemica (Belgium), dried with calcium hydride for $24 \mathrm{~h}$, and distilled under vacuum $\left(82^{\circ} \mathrm{C} / 133 \mathrm{~Pa}\right)$. Stannous octoate (analytical grade) and isoniazid (INH, 99\%) were purchased from Sigma-Aldrich. RFP was purchased from Energy Chemical, China. $\beta$-tricalcium phosphate $(\beta$-TCP) powder with diameter of 300-500 nm was purchased from the Forth Reagent Factory of Shanghai, China. Ethyl acetate was dried by $\mathrm{P}_{2} \mathrm{O}_{5}$ overnight and then distilled. $\mathrm{NaCl}$ of analytical quality was purchased from Beijing Chemical Works, China. It was sieved and particles with diameter of $150-300 \mu \mathrm{m}$ were selected. Gelatin (analytical grade), solvents and other compounds were obtained from Beijing Chemical Reagents Company, China. All reagents were used as received unless otherwise noted.

2.2. Characterizations. ${ }^{1} \mathrm{H}$ NMR spectra was obtained on a Bruker DRX-400 spectrometer in chloroform-d using tetramethylsilane (TMS) as an internal reference. Gel permeation chromatography (GPC) measurements were carried out on VE-2001 (Viscotek, UK) maintained at $35^{\circ} \mathrm{C}$ using chloroform as eluent at a flow rate of $1.0 \mathrm{~mL} / \mathrm{min}$. Scanning electron microscopy (SEM) images were obtained at acceleration voltage of $5 \mathrm{kV}$ on a JSM-6700F microscope (JEOL, Japan). The samples were sputter-coated with a thin layer of $\mathrm{Pt}$ for $120 \mathrm{~s}$ to make the samples conductive before testing. Thermogravimetric analysis (TGA) measurements were performed on a TA Instruments, Inc., MDSC-2910. The temperature program was from 30 to $700^{\circ} \mathrm{C}$ with an increasing rate of $10^{\circ} \mathrm{C} \mathrm{min}^{-1}$ in a flow of air. UV-vis spectra were measured on a Hitachi U-3010 spectrometer and fluorescence measurements were carried out on a Hitachi F4600 photoluminescence spectrometer with a xenon lamp as a light source. Confocal laser scanning microscopy (CLSM, Zeiss LSM 510, Germany) was under excitation at $314 \mathrm{~nm}$.

2.3. Preparation and Characterization of $b$-PLGC. According to our previous report, poly( $\varepsilon$-caprolactone)-block-poly(lacticco-glycolic acid) (b-PLGC) copolymer was synthesized via two steps [15]. Firstly, the PCL pre-polymer was prepared by ringopening polymerization of $\varepsilon$-caprolactone (CL) under $65 \mathrm{~Pa}$ in sealed glass ampoules at $140^{\circ} \mathrm{C}$ for $20 \mathrm{~h}$ in the presence of hexadecanol as initiator and stannous octoate as catalyst $(0.05$ wt\%). Subsequently, purified PCL pre-polymer, glycolide, lactide, and stannous octoate were sealed into a deoxygenating glass tube under vacuum at $160^{\circ} \mathrm{C}$ for $20 \mathrm{~h}$. After that, the raw product was dissolved in chloroform, precipitated into ethanol and then dried thoroughly under vacuum for $48 \mathrm{~h}$.

\subsection{Preparation and Characterization of Gelatin INH-Loaded}

Particles. INH-loaded Gelatin particles were prepared via a 
modified O/W emulsion technique [27]. Firstly, INH solution with concentration of $10 \mathrm{wt} \%$ was made by dissolving INH in deionized water. Then a certain amount of TCP nanoparticles were added into. After the TCP nanoparticles were evenly dispersed, $1.0 \mathrm{~g}$ of gelatin was put into the $10 \mathrm{~mL}$ of $\mathrm{INH}$ solution containing TCP nanoparticles. The solution was heated to $45^{\circ} \mathrm{C}$ as the water phase, which was slowly added to the $350 \mathrm{~mL}$ of soybean oil containing the $0.675 \mathrm{~g}$ of span 80 as oil phase with stirring speed of $200 \mathrm{rpm} .10$ minutes later, the stirring speed was raised up to $400 \mathrm{rpm}$ and further kept for $30 \mathrm{~min}$ while the system was naturally cooling down to room temperature. After that, the product was washed by acetone for 4 times and isopropanol for 3 times. Next, the obtained microparticles were freeze-dried for $48 \mathrm{~h}$ and then crosslinked by the glutaraldehyde dissolved in $100 \mathrm{~mL}$ of acetone. Afterwards, the particles were added into $300 \mathrm{~mL}$ of solution with $10 \mathrm{mmol}$ glycine and $0.1 \mathrm{wt} \%$ span 80 to remove the residual glutaraldehyde. At last, the microparticles were washed by water for 3 times. After the freeze-dried process for $48 \mathrm{~h}$, the final samples were sealed and kept in a $4^{\circ} \mathrm{C}$ refrigerator. The microparticles with theoretical TCP content of $0 \mathrm{wt} \%, 33 \mathrm{wt} \%$ and $50 \mathrm{wt} \%$ were abbreviated as INH@GMs, INH-TCP@GMs3367 and INH-TCP@GMs5050, respectively.

After the microparticles were coated with platinum using a sputter coater (E-1010, Hitachi Ltd, Japan), their morphology was observed by SEM (JSM-6700F, JEOL Ltd, Japan). The average diameter was calculated by measuring 5 SEM pictures of each sample with more than 100 particles on every picture measured. TCP content was measured by TGA (Pyris 1 , PerkinElmer, USA) with a temperature range of $30-700^{\circ} \mathrm{C}$ and a temperature rise speed of $10^{\circ} \mathrm{C} / \mathrm{min}$.

\subsection{Preparation and Characterization of Composite} Scaffolds. Based on our previous study, the composite scaffolds were fabricated by particle leaching combined with phase separation techniques [15, 24, 25]. The b-PLGC scaffolds loaded with INH-TCP@GMs and RFP were prepared as follows: Firstly, b-PLGC, RFP and 1,4-dioxane in the proportion of 5: 1:90 (wt. ratio) were stirred for $24 \mathrm{~h}$ to form a solution. Then a certain quality of INH-TCP@GMs5050 and pre-sieved $\mathrm{NaCl}$ particles with a weight ratio of $40 / 1$ to $b$-PLGC were added into the solution and stirred vigorously to form a slurry mixture. Next, the slurry was fitted into a mold, frozen under $-20^{\circ} \mathrm{C}$ and freeze-dried for $48 \mathrm{~h}$. After a complete rinse with distilled water and a subsequent $24 \mathrm{~h}$ freeze-drying, the PLGC scaffolds loaded with INH and RFP was obtained. The content of INH-TCP@GMs5050 in the obtained scaffolds were $50 \mathrm{wt} \%$ so these scaffolds were abbreviated as I-M/RP50.

The $b$-PLGC scaffolds loaded with INH-TCP@GMs was prepared similarly, and the only difference was that no RFP was introduced in the $b$-PLGC solution. The contents of INHTCP@GMs5050 in the scaffolds were $50 \mathrm{wt} \%$ and $70 \mathrm{wt} \%$, so these scaffolds were marked as I-M/P50 and I-M/P70, respectively.

The b-PLGC scaffolds loaded with INH and without INHTCP@GMs were prepared likewise, and the only difference was that TCP nanoparticles and INH were respectively added into the $b$-PLGC solution directly to take the place of INHTCP@GMs5050. The INH-loaded $b$-PLGC scaffold without
INH-TCP@GMs was named as INH/TCP/b-PLGC for short. After the scaffolds were coated with platinum using a sputter coater, their morphology was characterized by SEM. The scaffolds carrying gelatin particles loaded with Evans blue and TCP were cut into slices and observed under a confocal laser scanning microscopy (CLSM, Zeiss LSM 510, Germany) at $540 \mathrm{~nm}$. Pore size was measured using the ImageJ software (National Institutes of Health, USA) according to the SEM micrographs. For each scaffold, the average diameter of the pores was calculated based on 100 measurements. The porosity of the scaffolds was determined as described previously [28]. TCP content of scaffolds was measured by TGA (Pyris 1, PerkinElmer, USA) with a temperature range of $30-700^{\circ} \mathrm{C}$ and a temperature rise speed of $10^{\circ} \mathrm{C} / \mathrm{min}$.

\subsection{INH Loading Content and Entrapment Efficiency of} Gelatin Particles. INH Loading content and entrapment efficiency of gelatin particles were confirmed by extraction method. Firstly, a standard curve was gained by a series of INH concentrations in PBS using a UV spectrophotometer (TU-1901, PERSEE, China) at $\lambda_{\max }=263 \mathrm{~nm}$. Then $10 \mathrm{mg}$ of INH-loaded particles was added into $\mathrm{NaOH}$ solution and the solution was heated to $60^{\circ} \mathrm{C}$ for $2 \mathrm{~h}$ to dissolve particles completely. Next, hydrochloric acid was put in to neutralize the alkalinity and dilute PBS to $5 \mathrm{~mL}$. After that, the solution was centrifuged at $8000 \mathrm{rpm}$ for $10 \mathrm{~min}$ to remove the TCP particles and acquire clear solution, which was analyzed using a UV spectrophotometer at $\lambda_{\max }=263 \mathrm{~nm}$. Meanwhile, the same quality of pure particles without INH was treated in the same way as the INH-loaded particles and the clear solution obtained were served as a control. The INH concentration was calculated through a calibration curve gained from INH standard solutions at different concentrations. The percentages of INH loading and encapsulation efficiency were calculated as follows:

INH loading content $(\%)=\frac{(\text { Weight of INH in particle })}{(\text { Weight of INH-loaded particle }) \times 100 \%}$,

INH entrapment efficiency $(\%)=\frac{(\text { Weight of INH in particle })}{(\text { Weight of INH feeding }) \times 100 \%}$.

2.7. INH Release Behaviours of Particles. In vitro release behaviours of INH@GMs, INH-TCP@GMs3367 and INH-TCP@GMs5050 were carried out at $37^{\circ} \mathrm{C}$ in $10 \mathrm{~mL}$ of $0.1 \mathrm{M}$ PBS ( $\mathrm{pH}=7.4)$, respectively. The release medium was withdrawn at pre-determined time intervals and replaced with fresh PBS each time. Then the INH was determined by the UV spectrophotometer at $\lambda_{\max }=263 \mathrm{~nm}$. The concentration of INH was calculated with a calibration curve from INH standard solutions at different concentrations.

2.8. Drug Release Behaviours of Various Scaffolds. In vitro release behaviours of various scaffolds were performed at $37^{\circ} \mathrm{C}$ in $10 \mathrm{~mL}$ of $0.1 \mathrm{M} \mathrm{PBS}(\mathrm{pH}=7.4)$, respectively. The release medium was taken away at pre-set time intervals and displaced with fresh PBS each time. Then the INH and RFP were determined by UV spectrophotometer at $\lambda_{\max }=263 \mathrm{~nm}$ 


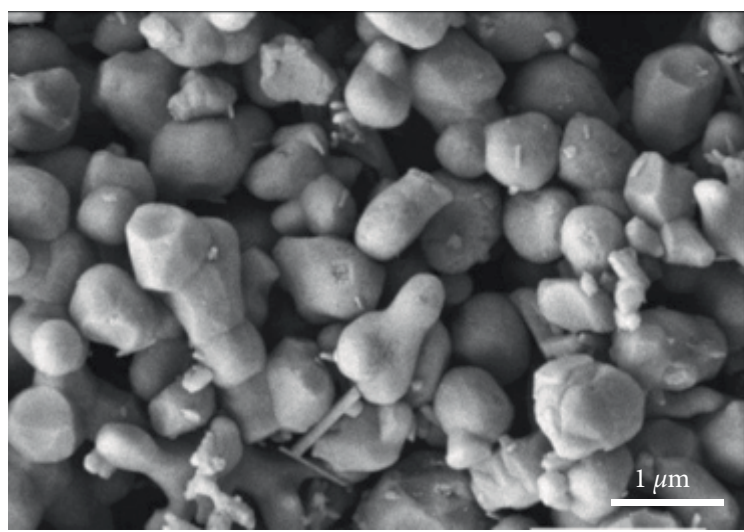

(a)

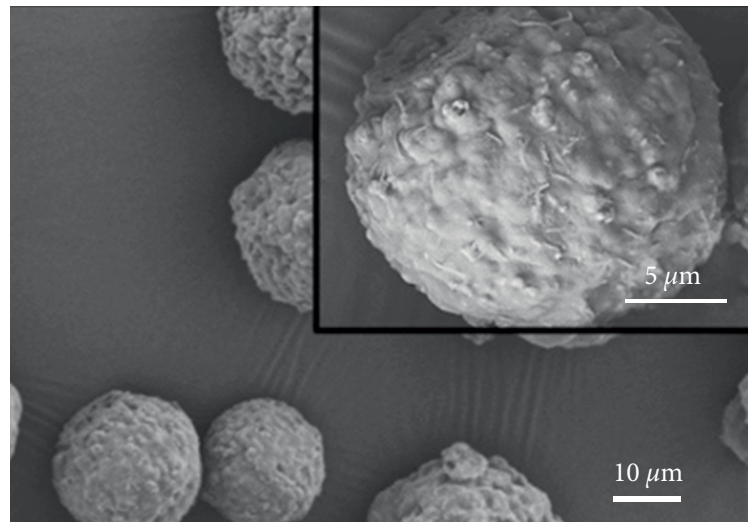

(c)

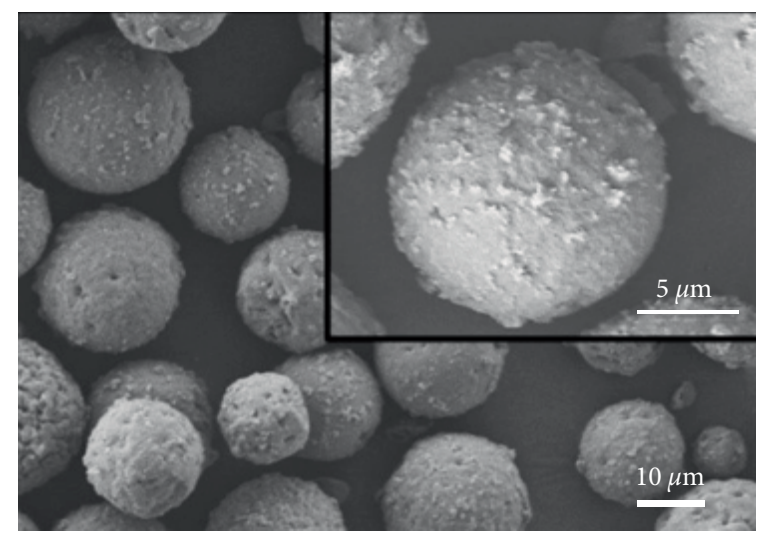

(b)

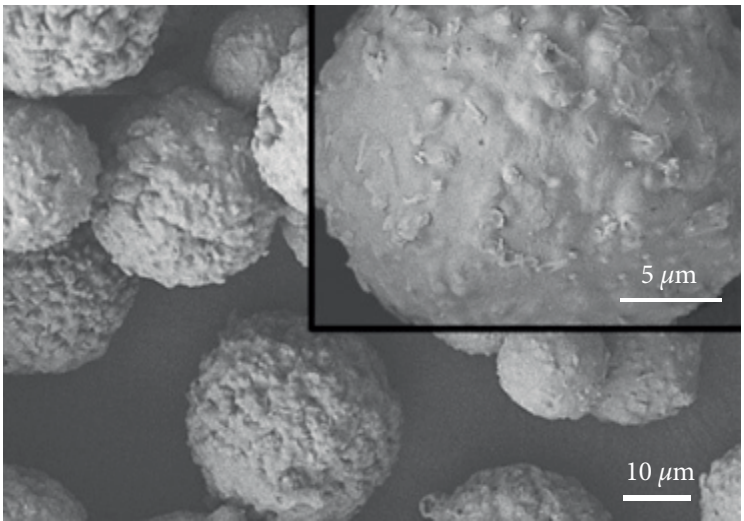

(d)

FIGURE 1: SEM images showing the TCP and INH-loaded gelation microparticles of (a) TCP, (b) INH@GMs, (c) INH-TCP@GMs3367 and (d) INH-TCP@GMs5050.

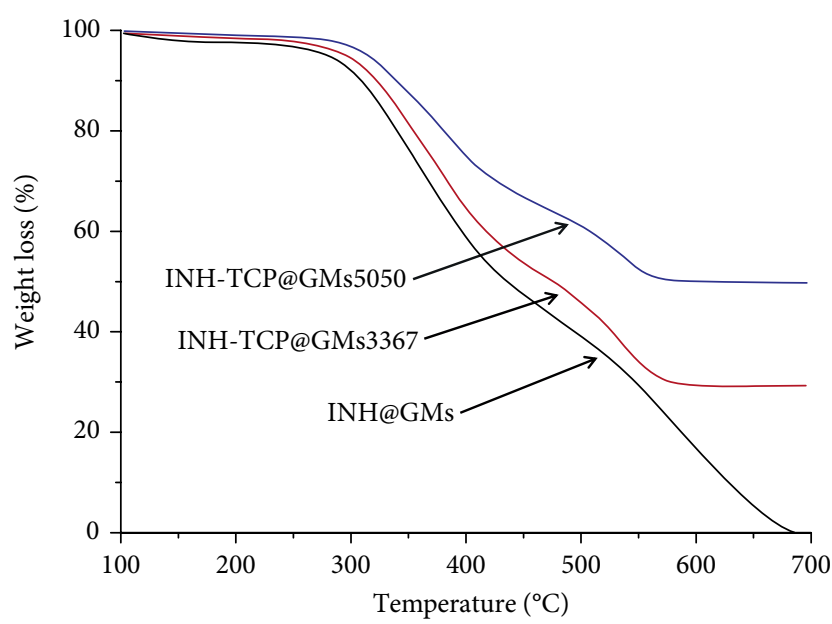

FIGURE 2: TGA curves of various INH-loaded microparticles.

and $474 \mathrm{~nm}$, respectively. The contents of INH and RFP were calculated with the calibration curve from INH and RFP standard solutions at different concentrations.

2.9. Statistical Analysis. All quantitative data were expressed as mean \pm standard deviation $(n=3)$. Statistical analysis was made based on $t$-test and the differences.
TABLE 1: INH loading content and entrapment efficiency of GMs and TCP@GMs microparticles.

\begin{tabular}{lccc}
\hline Parameters & GMs & $\begin{array}{c}\text { Microparticles } \\
\text { TCP@ } \\
\text { GMs3367 }\end{array}$ & $\begin{array}{c}\text { TCP@ } \\
\text { GMs5050 }\end{array}$ \\
\hline $\begin{array}{l}\text { INH loading } \\
\text { content (wt\%) }\end{array}$ & $3.7 \pm 0.2$ & $4.5 \pm 0.3$ & $5.0 \pm 0.5$ \\
$\begin{array}{l}\text { Entrapment } \\
\text { efficiency (\%) }\end{array}$ & $37.0 \pm 2.0$ & $45 \pm 3.0$ & $50 \pm 5.0$ \\
\hline
\end{tabular}

${ }^{\mathrm{a}}$ Feeding dose of INH to (TCP + GM) in GMs and TCP@GMs microparticles was 10:90.

\section{Results and Discussion}

\subsection{Fabrication of b-PLGC Scaffolds Loaded with INH and RFP}

3.1.1. Preparation and Characterization of the INH-Loaded Gelatin Microparticles. The morphology of INH-loaded gelatin microspheres was prepared by the modified O/W emulsion technique. As shown in Figure 1, SEM images showed the particle size of TCP with a range of 300-500 nm while the size of INH-loaded gelatin particles was $10-30 \mu \mathrm{m}$. As the increase of TCP content, the particle sizes of INH@ GMs, INH-TCP@GMs3367 and INH-TCP@GMs5050 were 

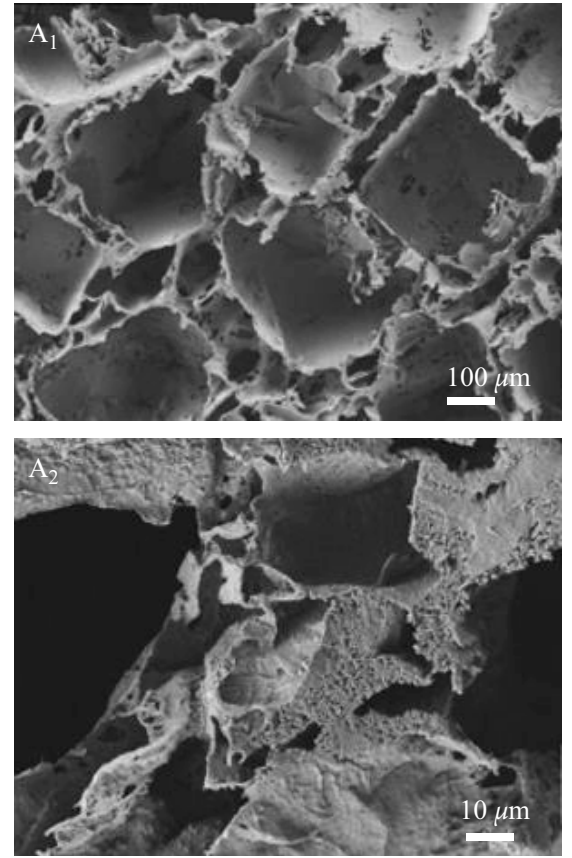

(a)
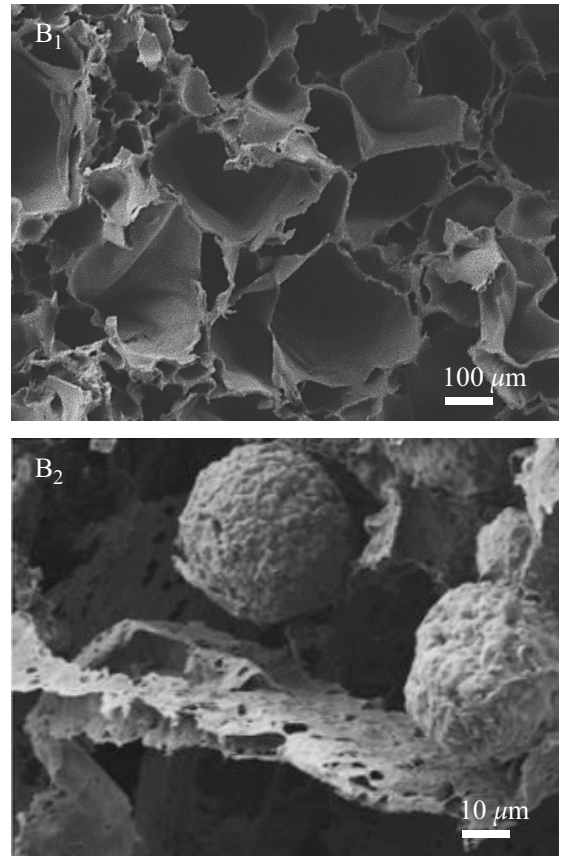

(b)
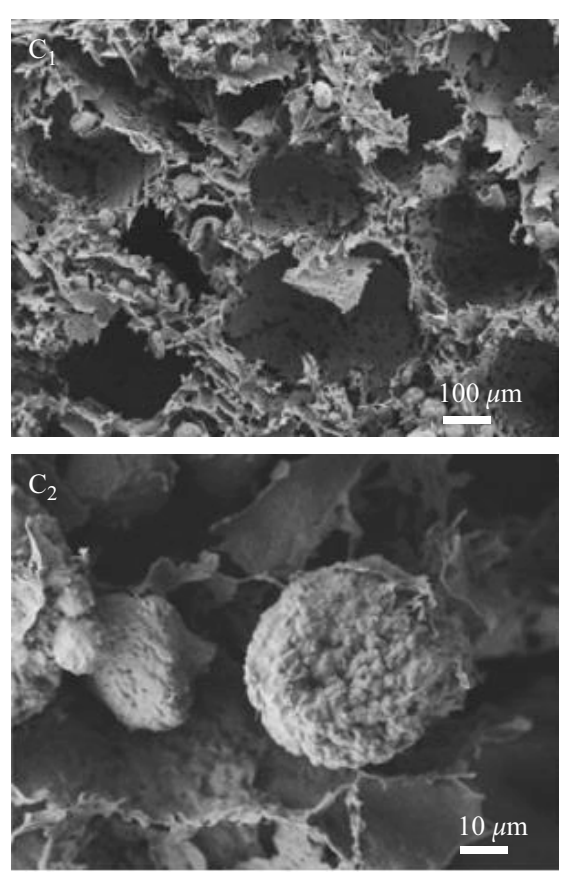

(c)

FIGURE 3: SEM images showing the INH-loaded $b$-PLGC scaffolds with different contents of microparticles. (a) INH/TCP/ $b$-PLGC scaffolds; (b) I-M/P50 scaffolds and (c) I-M/P70 scaffolds.

TABLE 2: Pore parameters of INH-loaded $b$-PLGC scaffolds with different contents of microparticles.

\begin{tabular}{lccc}
\hline & \multicolumn{3}{c}{ Scaffolds } \\
Parameters & $\begin{array}{c}\text { INH/TCP/b- } \\
\text { PLGC }\end{array}$ & I-M/P50 & I-M/P70 \\
\hline $\begin{array}{l}\text { Average pore } \\
\text { diameter }(\mu \mathrm{m})\end{array}$ & $253.0 \pm 34.0$ & $234.0 \pm 39.0$ & $231.0 \pm 27.0$ \\
Porosity $(\%)$ & $87.6 \pm 1.5$ & $84.5 \pm 2.1$ & $83.8 \pm 1.4$ \\
\hline
\end{tabular}

$17.2 \pm 2.1 \mu \mathrm{m}, 21.8 \pm 1.9 \mu \mathrm{m}$ and $23.1 \pm 2.4 \mu \mathrm{m}$ obtained from ImageJ software, respectively.

The TCP content in the microparticles was measured by thermogravimetric analysis (TGA) as showed in Figure 2. The quantities of INH@GMs, INH-TCP@GMs3367 and INHTCP@GMs5050 were 0 wt $\%, 29.3$ wt $\%$ and 50.0 wt $\%$ respectively, which were conformed to their theoretical contents, indicating the minimal loss of TCP in the preparation of microparticles.

As shown in Table 1, the INH loading content and entrapment efficiency of various microparticles were ranged from $3.7 \%$ to $5.0 \%$ and $37 \%$ to $50 \%$, respectively. When the TCP content was increased, the INH loading content and entrapment efficiency grew correspondingly, which was attributed to the relatively large surface area of TCP that possessed a certain drug absorption effect. However, there was still a part of the drug loss during the microparticles preparing process due to mechanical agitation and slight solubility of INH in acetone. The above results manifested the successful preparation of the INH-loaded gelatin microparticles.

\subsection{Preparation and Characterization of $b$-PLGC Scaffolds} Loaded with INH-TCP@GMs and RFP. The $b$-PLGC scaffolds loaded with INH-TCP@GMs were fabricated by particle leaching combined with phase separation technique. The morphology of scaffolds was determined by SEM observation and compared with the $b$-PLGC scaffolds loaded INH without microparticles, as shown in Figure 3. The pore size of the scaffolds loaded with INH-TCP@GMs was in a range of 200-300 $\mu \mathrm{m}$. Similar with the INH/TCP/b-PLGC scaffolds, numerous micro-pores $(10-50 \mu \mathrm{m})$ could be seen on the wall of macro-pores. The results manifested that the microparticles did not affect the characterization of scaffolds, which could provide a high surface area for the cell seeding, attachment and proliferation, deliver nutrient and elimination of metabolized products [29-32].

The pore size and porosity of scaffolds were also acquired respectively by dint of ImageJ software and volume exclusive method as shown in Table 2. The pore sizes of I-M/P50 and I-M/P70 scaffolds were in the range of $200-300 \mu \mathrm{m}$ while the porosity was above $80 \%$. These results pointed out that the pore size and porosity of scaffolds were slightly decreased after incorporating the INH-TCP@GMs.

To further analyze the distribution of microparticles, a water-soluble fluorescent polymer, Evans blue, was loaded on the microparticles instead of INH. The formed composite scaffolds, described as E-M/P50 and E-M/P70, were analyzed by the confocal laser scanning in Figure 4. The results showed that these microparticles were distributed throughout these scaffolds with a relative uniformity, e.g. some particles spread at the junction of different pores and others were embedded in the inner of scaffolds. 

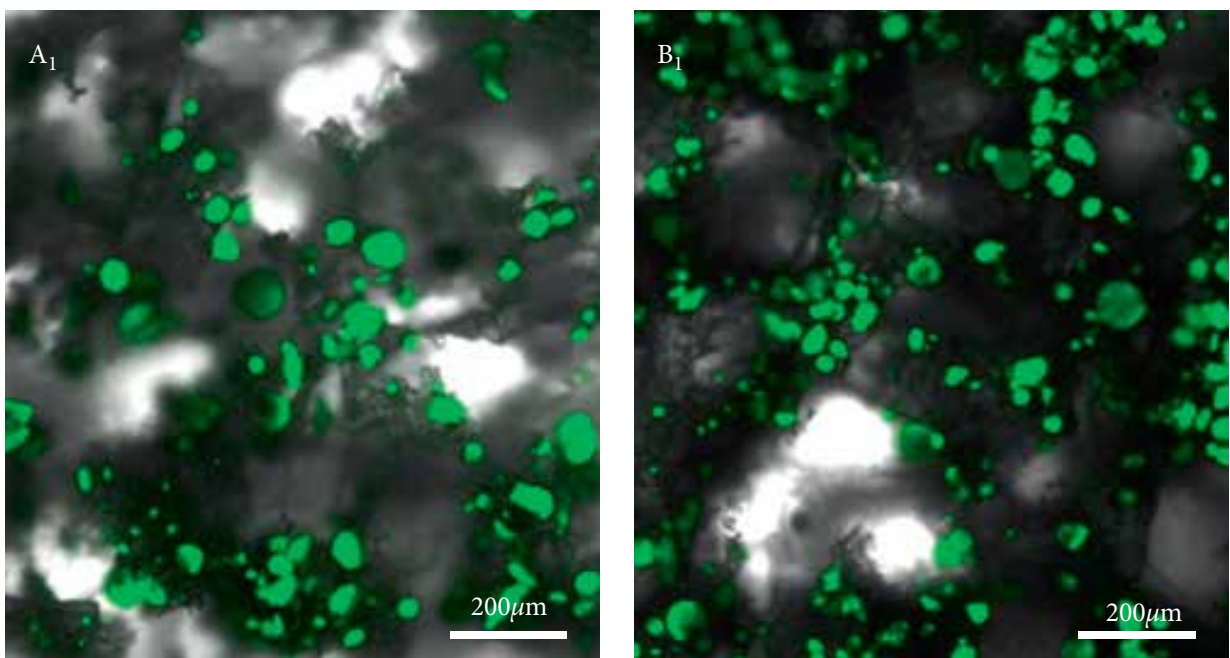

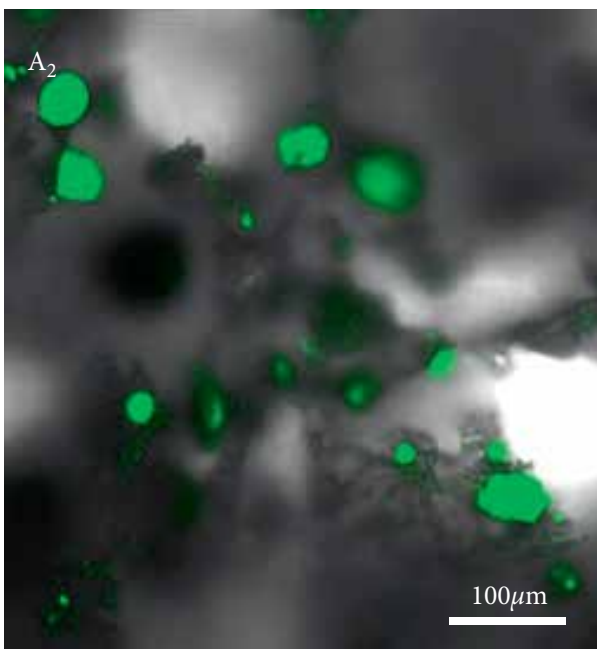

(a)

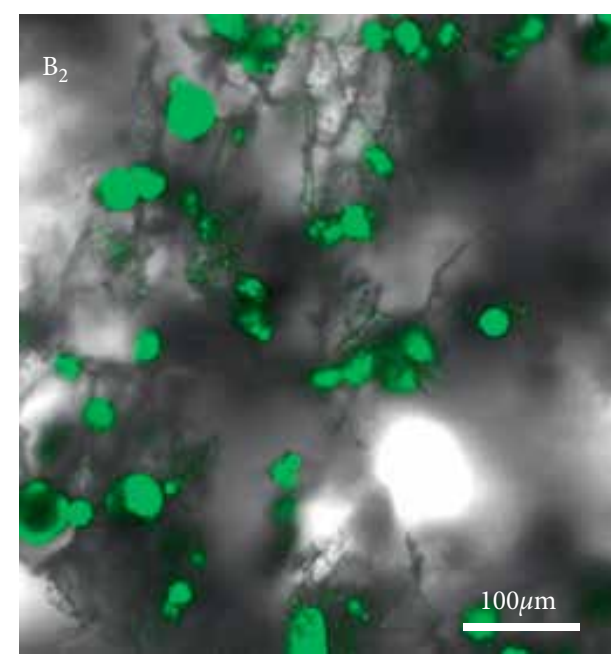

(b)

FIGURE 4: CLSM images of Evans blue loaded TCP@GMs/ $b$-PLGC scaffolds of (a) E-M/P50 and (b) E-M/P70.

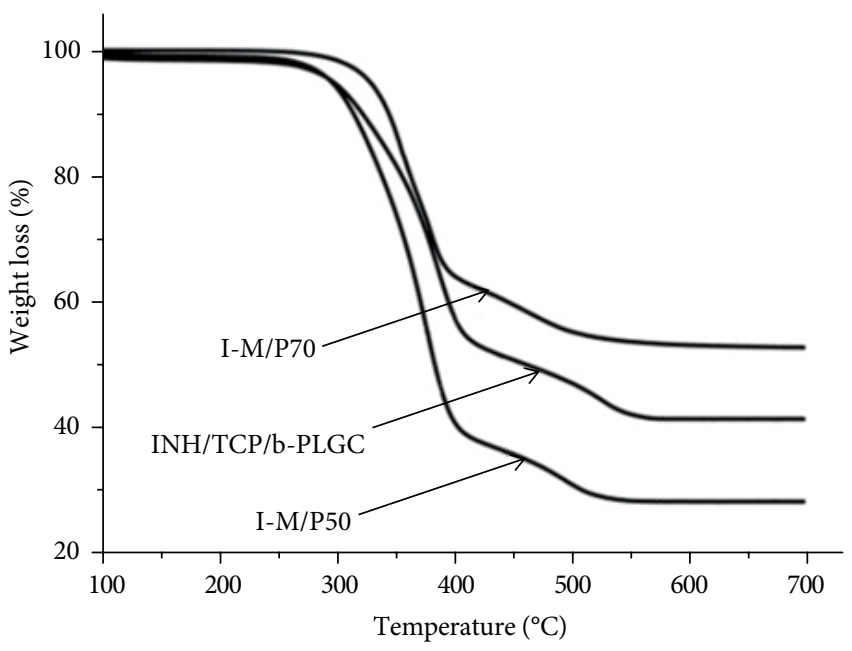

(a)

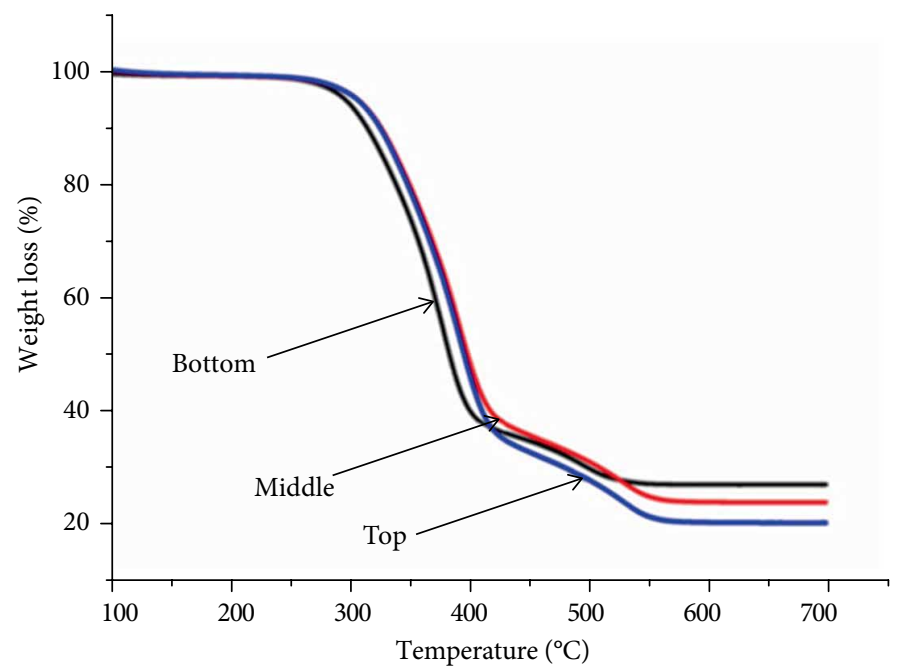

(b)

FIGURE 5: TGA curves of INH-loaded $b$-PLGC scaffolds with (a) different content of microparticles and (b) different parts of I-M/P50 scaffolds. 


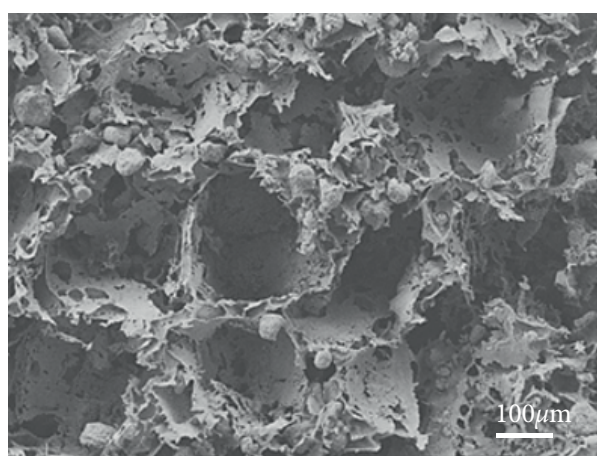

(a)

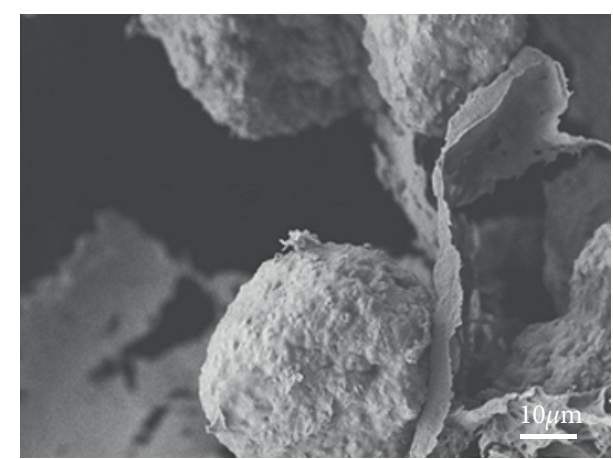

(b)

FIGURE 6: Morphologies of I-M/RP50 scaffolds.

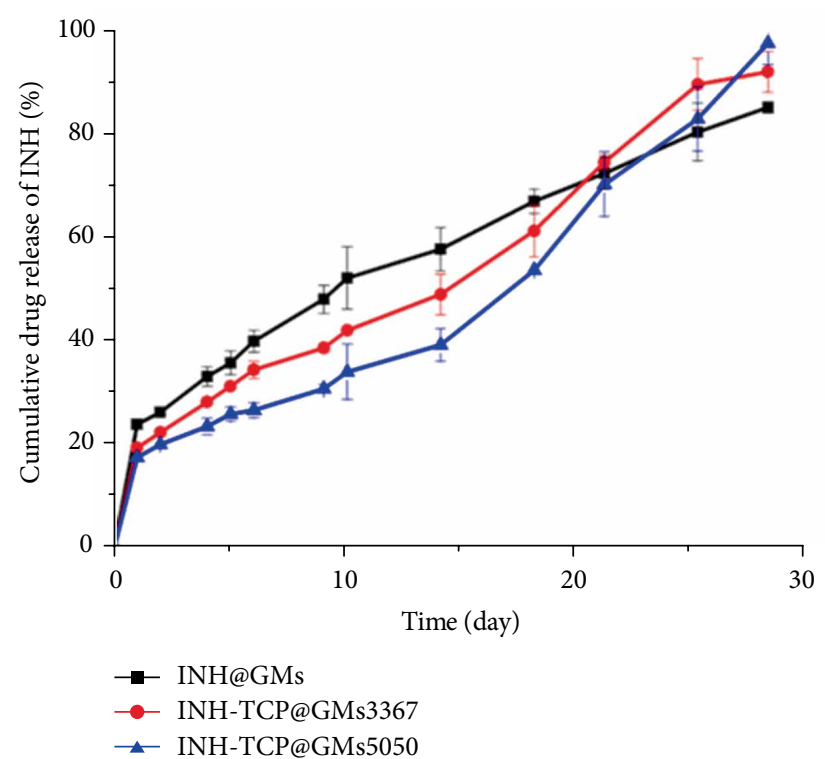

FIGURE 7: Release behaviours of INH@GMs and INH-TCP@GMs in vitro.

The TCP contents of the scaffolds were also in keeping with the theoretical values with the verification by the TGA measurement (Figure 5(a)), indicating the minimal TCP loss during preparation of scaffolds. Since the TCP distribution in scaffolds exerted an influence over bone regeneration, the TCP content of I-M/P50 scaffolds in different parts was also measured by TGA (Figure 5(b)). At the top, middle and bottom parts the content was $21.2 \mathrm{wt} \%, 24.7 \mathrm{wt} \%$ and $27.8 \mathrm{wt} \%$, respectively. The trivial distribution difference of TCP content in the whole scaffolds may be ascribed to a bit of sedimentation of microparticles during the preparing process of scaffolds, but it would not impact the usage of scaffolds.

The $b$-PLGC scaffolds were loaded with INH-TCP@GMs and RFP according to the previous methods [15]. Compared to the I-M/P50, I-M/RP50 was also in possession of random interconnected porous structures in Figure 6. Its pore size was in a range of $200-300 \mu \mathrm{m}$ along with numerous micro-pores $(10-50 \mu \mathrm{m})$ on the wall of macro-pores. Besides, the pore porosity and TCP content of the I-M/RP50 scaffolds were also similar to the I-M/P50 scaffolds (the corresponding data and curves were not shown to avoid repetition). These results manifested that the RFP had no obvious influence on the morphology of scaffolds.

\subsection{Drug Release Behaviours of b-PLGC Scaffolds Loaded with INH and RFP In Vitro}

3.2.1. INH Release Behaviours of Microparticles In Vitro. The initial burst releases of INH@GMs, INH-TCP@GMs3367 and INH-TCP@GMs5050were 24.4wt\%, 19.8 wt\% and 17.9 wt\% respectively in Figure 7. As the TCP content was increased, the burst release fell down, indicating that TCP could inhibit the initial burst release to some extent. After the initial burst release, INH release behaviours of these three microparticles were essentially uniform with linear trends. The release rate diminished as the TCP content augmented. But the drug release speed of INH-TCP@GMs accelerated after three weeks of inhibition effect, especially for the INH-TCP@GMs5050 whose cumulative release reached $98.2 \%$ on the fourth week, higher than 92.7\% in INH-TCP@GMs3367 and 85.7\% in INH@GMs.

After 28 days, the morphologies of microparticles were observed Figure 8. Obviously, gelatin microparticles without TCP were still able to maintain their spherical shape while the majority of those particles containing TCP were degraded as well as the exposure of TCP on the surfaces. The higher contents of TCP could cause the quicker degradation for these microparticles, which was consistent with the drug release behaviours after 3 weeks. Based on the above results of high INH entrapment efficiency, slow initial drug release rate and thorough ultimate drug release content, INH-TCP@GMs5050 was an ideal material for fabricating the INH loaded $b$-PLGC scaffolds.

\subsubsection{Drug Release Behaviours of INH Loaded b-PLGC Scaffolds} In Vitro. Drug release behavior of INH loaded $b$-PLGC scaffolds is shown in Figure 9(a). The initial INH burst release of INH/TCP/b-PLGC was higher and the cumulative INH release in the first week exceeded $60 \%$, but that of I-M/P50 and I-M/P70 scaffolds was less than $20 \%$. The sustained INH release of I-M/P50 and I-M/P70 scaffolds could keep for 84 days, and the cumulative release increased with the climbing content of INH-TCP@GMs5050 in scaffolds. The rapid INH 

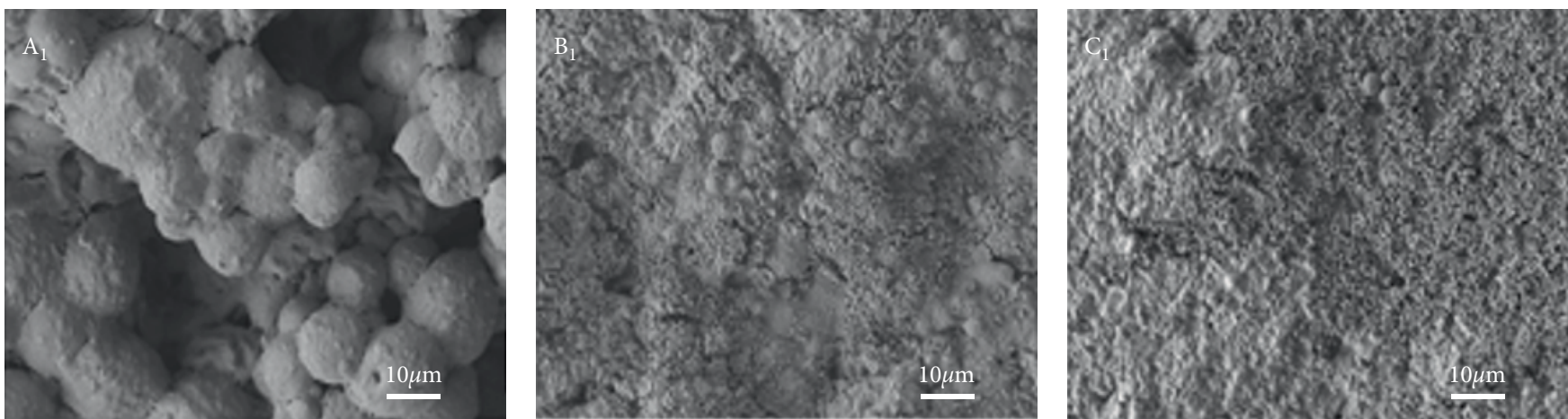

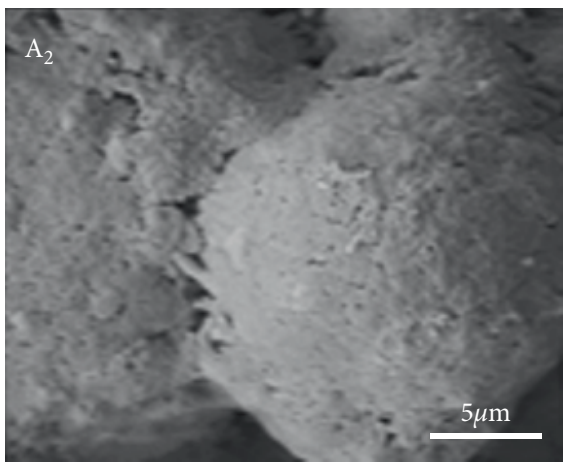

(a)

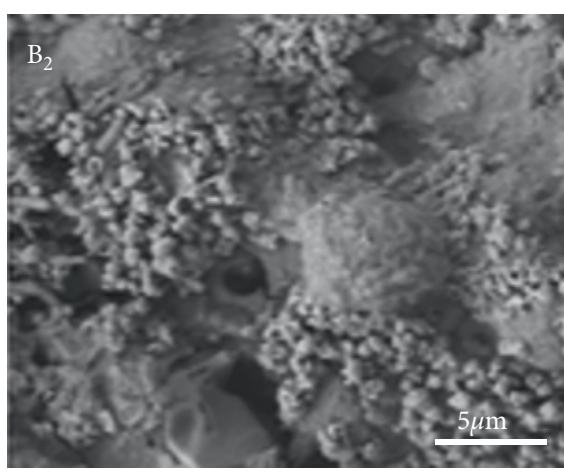

(b)

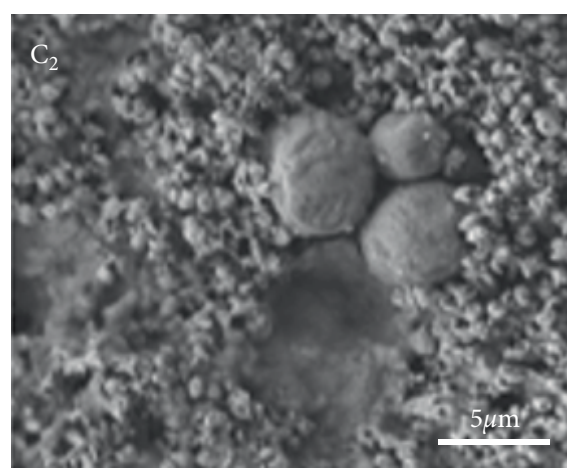

(c)

FIGURE 8: SEM images showing the GMs and TCP@GMs after release for 28 days. (a) GMs; (b) TCP@GMs3367, and (c) TCP@GMs5050.

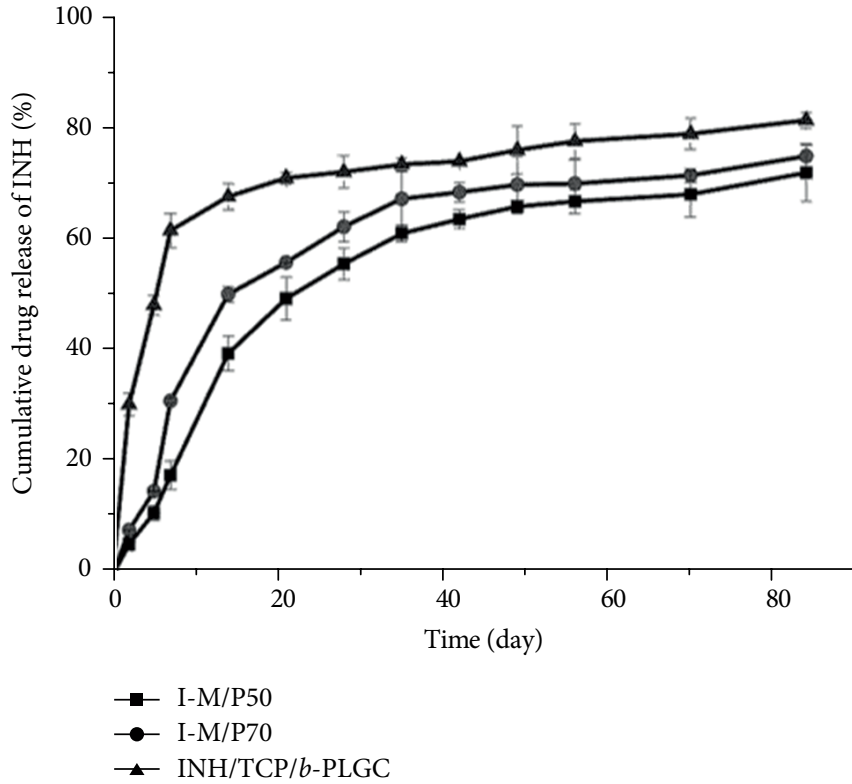

(a)

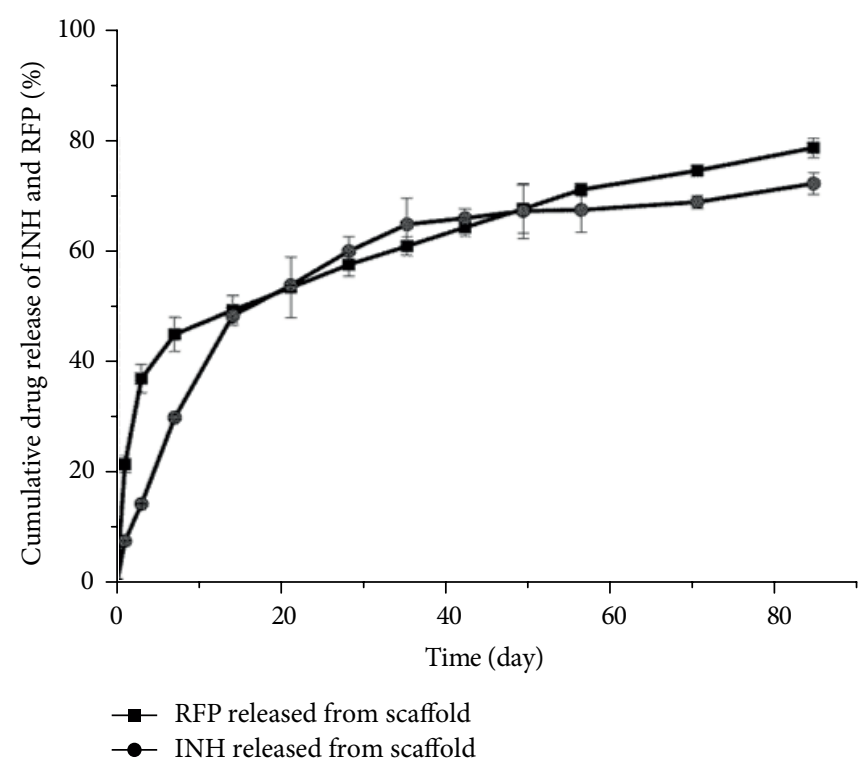

(b)

FIGURE 9: (a) Release behaviours of INH-loaded scaffolds in vitro. (b) INH and RFP Release behaviors of I-M/RP50 scaffolds in vitro.

release of INH/TCP/b-PLGC should be attributed to that of the water-soluble INH was directly blended in the scaffolds that were easy to contact with water. The sustained INH release of $b$-PLGC scaffolds loaded with INH-TCP@GMs5050 was mainly caused by the sustained INH release of INH-TCP@ GMs5050. In addition, since microparticles were coated with polymers during scaffold preparation process, some particles were embedded in the inner of scaffolds (Figure 5) and the INH was prevented to be directly in touch with water, resulting in the decreasing initial burst release and prolonging the release time. It is mentioned that this dual drugs-loaded system could achieve the expectant roles of respective drugs without obvious influences with each other.

The INH and RFP release profiles of I-M/RP50 scaffolds were shown in Figure 9(b). At the initial stage, a slight INH and RFP initial burst release occurred with about $15 \%$ and $20 \%$, respectively. After that, a long-term sustained INH and RFP release profiles were obtained along with the cumulative release of ca. $75 \%$ and $82 \%$ on day 84 , indicating that I-M/ RP50 scaffolds could carry out a sustained INH and RFP 
release for 3 months, which resembled the INH and RFP release behavior of individual INH-loaded (Figure 9(b)) and RFP-loaded scaffolds. The initial burst release of RFP should ascribe to inevitably existing RFP molecules on the surface of the substrate during the scaffold preparation. While the RFP molecules embedded inside polymeric matrixes would be slowly released via water molecule interpenetration and RFP diffusion effect. The INH and RFP release in vivo and its effect on repair and reconstruction of bone tissue would be further studied in our future work. In addition, there were still inevitably initial outbreak release problems that need to be improved. For example, we will design and fabricate another smart system to achieve the controllable varied-drugs delivery by further combining the supramolecular interactions and covalent bond methods.

\section{Conclusions}

In summary, we prepared a kind of INH-TCP@GMs microparticles with less initial burst release, higher cumulative release and longer sustained INH release, which was closely related to the TCP contents. After particle leaching combined with phase separation technique for the mixture of INH-TCP@GMs5050 and $b$-PLGC solution containing RFP, INH- and RFP-loaded b-PLGC scaffolds was obtained with the quicker INH release rate than INH-loaded $b$-PLGC scaffolds on account of the evenly distributed microparticles in these scaffolds. This drug release system could achieve their respective roles of two drugs, and the loading process was not affected with each other. After a slight initial burst release occurred on the first day, the INH and RFP were slowly released from the scaffolds for 84 days and the cumulative release achieved about $75 \%$ and $82 \%$ on day 84 , respectively. Thus, we believe that these dual anti-tuberculosis drug-loaded composite scaffolds will have potential clinical applications in bone tuberculosis therapy.

\section{Data Availability}

The data used to support the findings of this study are available from the corresponding author upon request.

\section{Conflicts of Interest}

The authors declare no conflict of interest.

\section{Authors' Contributions}

Dawei Li and Chao Li contributed equally to this work.

\section{Funding}

We greatly acknowledge the financial supports from the National Natural Science Foundation of China (NSFC, 81972081 and 21604093), the Beijing Novel Program (Z181100006218059) and the Open Research Project Funds (K2019-27).

\section{References}

[1] C. E. Dodd and L. S. Schlesinger, "New concepts in understanding latent tuberculosis," Current Opinion in Infectious Diseases, vol. 30, pp. 316-321, 2017.

[2] C. Shi and E. G. Pamer, "Monocyte recruitment during infection and inflammation," Nature Reviews Immunology, vol. 11, pp. 762-774, 2011.

[3] Y. R. Wang, Q. J. Wang, R. B. Zhu et al., "Trends of spinal tuberculosis research (1994-2015)," Medicine, vol. 95, no. 38, pp. 1994-2015, 2016.

[4] D. J. Short and T. Zgonis, "Management of osteomyelitis and bone loss in the diabetic charcot foot and ankle," Clinics in Podiatric Medicine and Surgery, vol. 34, no. 3, pp. 381-387, 2017.

[5] D. Li, L. T. Li, Y. L. Ma et al., "Dopamine-assisted fixation of drug-loaded polymeric multilayers to osteoarticular implants for tuberculosis therapy," Biomaterials Science, vol. 5, no. 4, pp. 730-740, 2017.

[6] C. E. Clancy, G. An, W. R. Cannon et al., "Multiscale modeling in the clinic: drug design and development," Annals of Biomedical Engineering, vol. 44, pp. 2591-2610, 2016.

[7] Y. J. Gao, Y. S. Ou, Q. X. Deng, B. He, X. Du, and J. Li, "Comparison between titanium mesh and autogenous iliac bone graft to restore vertebral height through posterior approach for the treatment of thoracic and lumbar spinal tuberculosis," PLoS One, vol. 12, no. 4, p. e0175567, 2017.

[8] J. Zou, Z. Shi, G. Mei, J. Xue, W. Gu, and X. Li, "Two-stage operation to treat destructive midfoot tuberculosis: 14 cases experience," Orthopaedics \& Traumatology: Surgery \& Research, vol. 102, pp. 1075-1080, 2016.

[9] M. Kalathur, S. Baiguera, and P. Macchiarini, "Translating tissue-engineered tracheal replacement from bench to bedside," Cellular and Molecular Life Sciences, vol. 67, no. 24, pp. 41854196, 2010.

[10] D. Huang, D. W. Li, T. T. Wang et al., "Isoniazid conjugated poly(lactide-co-glycolide): long-term controlled drug release and tissue regeneration for bone tuberculosis therapy," Biomaterials, vol. 52, pp. 417-425, 2015.

[11] M. L. Kang, J. K. Ko, J. E. Kim, and Gun-I. 1. Im, "Intra-articular delivery of kartogenin-conjugated chitosan nano/microparticles for cartilage regeneration," Biomaterials, vol. 35, pp. 9984-9994, 2014.

[12] R. Garcia-Alvarez, I. Izquierdo-Barba, and M. Vallet-Regi, “3D scaffold with effective multidrug sequential release against bacteria biofilm," Acta Biomaterialia, vol. 49, pp. 113-126, 2017.

[13] Y. G. Zhang, Y. J. Zhu, F. Chen, and T. W. Sun, "A novel composite scaffold comprising ultralong hydroxyapatite microtubes and chitosan: preparation and application in drug delivery," Journal of Materials Chemistry B, vol. 5, pp. 3898-3906, 2017.

[14] L. H. Nguyen, M. Y. Gao, J. Q. Lin, W. Wu, J. Wang, and S. Y. Chew, "Three-dimensional aligned nanofibers-hydrogel scaffold for controlled non-viral drug/gene delivery to direct axon regeneration in spinal cord injury treatment," Scientific Reports, vol. 7, p. 42212, 2017.

[15] P. Zhao, D. W. Li, F. Yang et al., "In vitro and in vivo drug release behavior and osteogenic potential of a composite scaffold based on poly(e-caprolactone)-block-poly(lactic-co-glycolic acid) and $\beta$-tricalcium phosphate," Journal of Materials Chemistry $B$, vol. 3, pp. 6885-6896, 2015.

[16] V. Kaushik, J. S. Yakisich, N. Azad et al., "Anti-tumor effects of cardiac glycosides on human lung cancer cells and lung 
tumorspheres," Journal of Cellular Physiology, vol. 232, pp. 2497-2507, 2017.

[17] S. Suzuki, T. Horinouchi, and C. Furusawa, "Acceleration and suppression of resistance development by antibiotic combinations," BMC Genomics, vol. 18, no. 1, 2017.

[18] Y. Zou, Y. Zhang, L. W. Han et al., "Oxidative stress-mediated developmental toxicity induced by isoniazide in zebrafish embryos and larvae," Journal of Applied Toxicology, vol. 37, no. 7, pp. 842-852, 2017.

[19] M. Njire, Y. J. Tan, J. Mugweru et al., "Pyrazinamide resistance in mycobacterium tuberculosis: review and update," Advances in Medical Sciences, vol. 61, no. 1, pp. 63-71, 2016.

[20] L. M. A. Alvarez, J. M. G. Garcia, M. D. P. Hernandez et al., "Utility of phenotypic and genotypic testing in the study of mycobacterium tuberculosis resistance to first-line antituberculosis drugs," Archivos de Bronconeumología, vol. 53, pp. 192-198, 2017.

[21] M. J. Chang, B. Jin, J. W. Chae et al., "Population pharmacokinetics of moxifloxacin, cycloserine, $\mathrm{p}$-aminosalicylic acid and kanamycin for the treatment of multi-drug-resistant tuberculosis," International Journal of Antimicrobial Agents, vol. 49, no. 6, pp. 677-687, 2017.

[22] L. F. Diniz, P. S. Carvalho, C. C. de Melo, and J. Ellena, "Development of a salt drug with improved solubility: ethionamide nitrate," Journal of Molecular Structure, vol. 1137, pp. 119-125, 2017.

[23] T. Maimakov, L. Sadykova, Z. Kalmataeva, K. Kurakpaev, and K. Šmigelskas, "Treatment of tuberculosis in south kazakhstan: clinical and economical aspects," Medicina, vol. 49, pp. 335-340, 2013.

[24] H. Shen, X. X. Hu, F. Yang, J. Bei, and S. Wang, "Cell affinity for bFGF immobilized heparin-containing poly(lactide-coglycolide) scaffolds," Biomaterials, vol. 32, pp. 3404-3412, 2011.

[25] F. Yang, W. J. Cui, Z. Xiong, L. Liu, and J. S. Bei Wang, "Poly(L, L-lactide-co-glycolide)/tricalcium phosphate composite scaffold and its various changes during degradation in vitro," Polymer Degradation and Stability, vol. 91, pp. 3065-3073, 2006.

[26] S. Khan, M. Ul-Islam, M. Ikram et al., "Three-dimensionally microporous and highly biocompatible bacterial cellulosegelatin composite scaffolds for tissue engineering applications," RSC Advances, vol. 6, pp. 110840-110849, 2016.

[27] H. Shen, Y. Niu, X. Hu, F. Yang, S. Wang, and D. Wu, "A biomimetic 3D microtubule-orientated poly(lactide-co-glycolide) scaffold with interconnected pores for tissue engineering," Journal of Materials Chemistry B, vol. 3, pp. 4417-4425, 2015.

[28] H. Shen, X. Hu, H. Cui et al., "Fabrication and effect on regulating vSMC phenotype of a biomimetic tunica media scaffold," Journal of Materials Chemistry B, vol. 4, pp. 76897696, 2016.

[29] A. Galperin, J. L. Thomas, and D. R. Buddy, "Degradable, thermo-sensitive poly( $\mathrm{N}$-isopropyl acrylamide)-based scaffolds with controlled porosity for tissue engineering applications," Biomacromolecules, vol. 11, no. 10, pp. 2583-2592, 2010.

[30] W. Teng, T. J. Long, Q. Zhang, K. Yao, T. T. Shen, and B. D. Ratner, "A tough, precision-porous hydrogel scaffold: ophthalmologic applications," Biomaterials, vol. 35, no. 32, pp. 8916-8926, 2014.

[31] H. K. Makadia and S. J. Siegel, "Polylactic-co-glycolic acid (PLGA) as biodegradable controlled drug delivery carrier," Polymers, vol. 3, pp. 1377-1397, 2011.
[32] F. J. O'Brien, B. A. Harley, I. V. Yannas, and L. J. Gibson, “The effect of pore size on cell adhesion in collagen-GAG scaffolds," Biomaterials, vol. 26, pp. 433-441, 2005. 


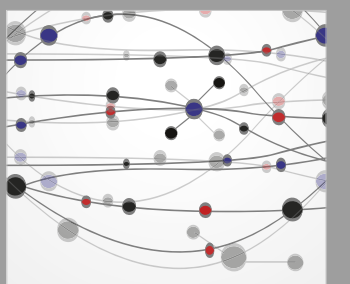

The Scientific World Journal
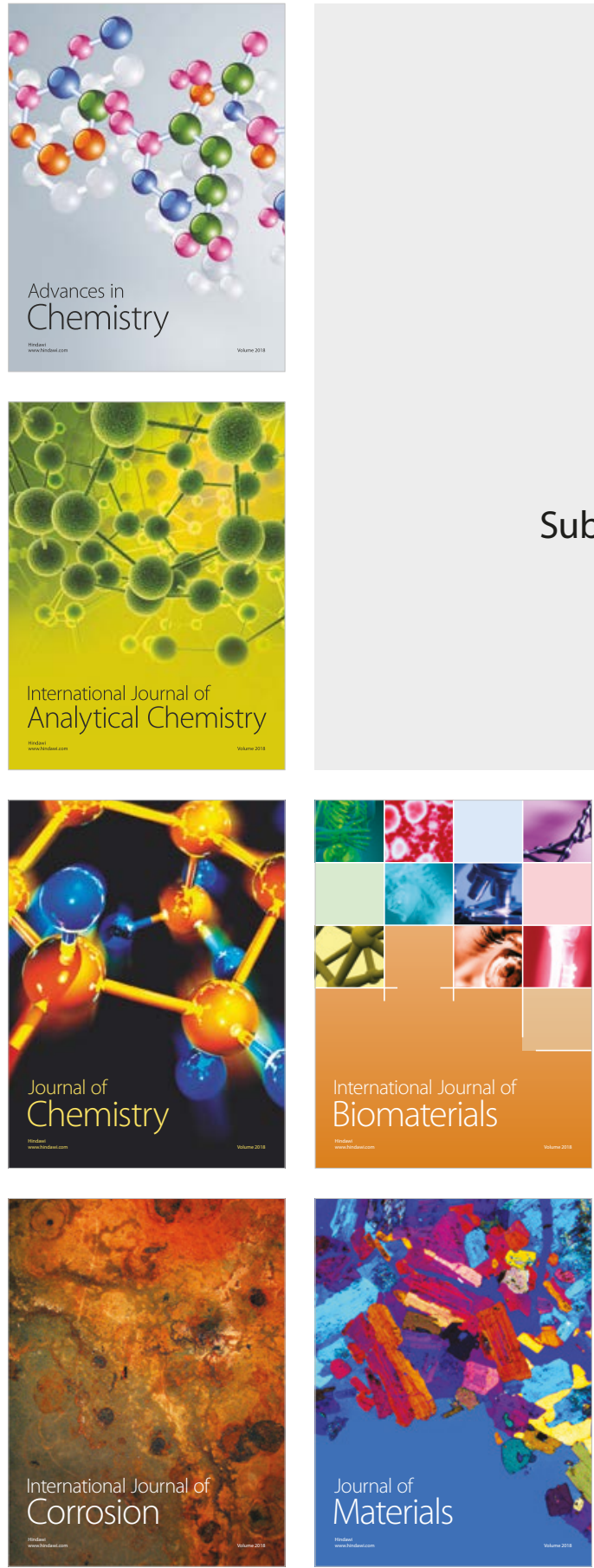

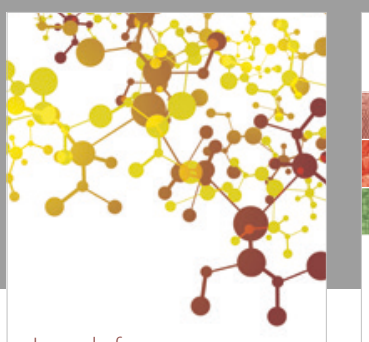

Journal of

Applied Chemistry
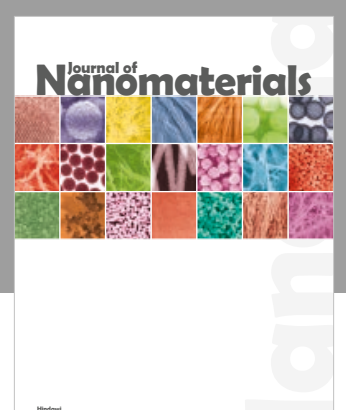

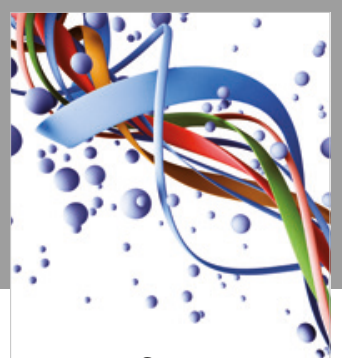

Scientifica

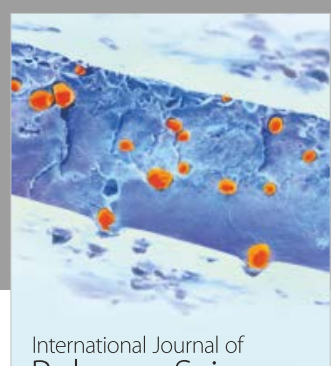

Polymer Science

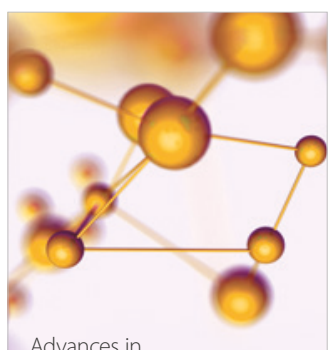

Physical Chemistry
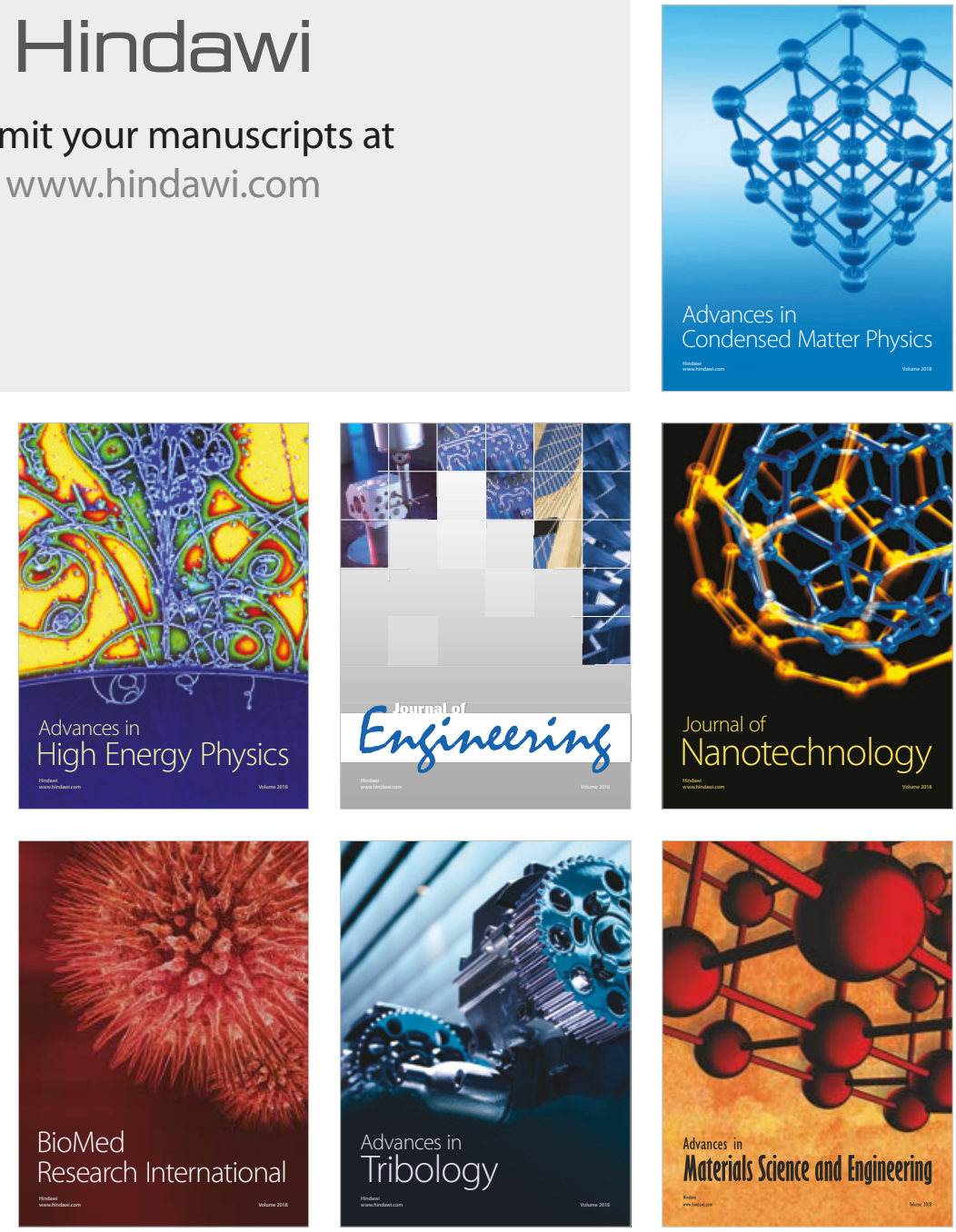